\title{
Long-term effects of the Chêneau brace on coronal and sagittal alignment in adolescent idiopathic scoliosis
}

\author{
Ming-Qiao Fang, MD, Chong Wang, MD, Guang-Heng Xiang, MD, Chao Lou, MD, \\ Nai-Feng Tian, PhD, and Hua-Zi Xu, MD \\ Zhejiang Spine Research Center, Department of Orthopaedic Surgery, Second Affiliated Hospital of Wenzhou Medical University, \\ Wenzhou, Zhejiang, China
}

OBJECT The aim of the present study was to retrospectively evaluate progressive correction of coronal and sagittal alignment and pelvic parameters in patients treated with a Chêneau brace.

METHODS Thirty-two patients with adolescent idiopathic scoliosis (AIS) were assessed before initiation of bracing treatment and at the final follow-up. Each patient underwent radiological examinations, and coronal, sagittal, and pelvic parameters were measured.

RESULTS No statistically significant modification of the Cobb angle was noted. The pelvic incidence remained unchanged in $59 \%$ of the cases and increased in $28 \%$ of the cases. The sacral slope decreased in $34 \%$ of the cases but remained unchanged in $50 \%$. Thoracic kyphosis and lumbar lordosis were significantly decreased, whereas the sagittal vertical axis was significantly increased from a mean of -44.0 to $-30.2 \mathrm{~mm}(p=0.02)$. The mean pelvic tilt increased significantly from $4.5^{\circ}$ to $8.3^{\circ}(p=0.002)$.

CONCLUSIONS The Chêneau brace can be useful for preventing curvature progression in patients with AIS. However, the results of this study reveal high variability in the effect of brace treatment on sagittal and pelvic alignment. Treatment with the Chêneau brace may also influence sagittal global balance.

http://thejns.org/doi/abs/10.3171/2015.2.SPINE14970

KEY WORDS Chêneau brace; adolescent idiopathic scoliosis; progressive correction; sagittal alignment; deformity

$\Lambda$ DOLESCENT idiopathic scoliosis (AIS) is characterized by lateral curvature of the spine with a Cobb angle of $>10^{\circ}$ and vertebral rotation. Treatment of AIS with a rigid brace, the most common nonoperative treatment for the prevention of curvature progression, is indicated for a defined group of patients based on their age, sex, magnitude of the curve, and pubertal status. ${ }^{6,12,17,18}$

The Chêneau brace, which is intended to de-rotate the apical segment of the curve and provide a 3D correction of the spinal deformity ${ }^{19}$ is the most widely used rigid thoracolumbosacral orthosis in Europe. ${ }^{2}$ The major mechanism of this orthosis is 3D correction of the spinal deformity through a system of multipoint pressure zones and expansion chambers. ${ }^{2,10}$ The Chêneau brace creates pressure on the convexity of a curve, and on the opposite side are wide expansion chambers in the frontal, sagittal, and horizontal planes. ${ }^{9}$ Regarding the outcomes of brace application, the Chêneau brace has been found to prevent curve progression and to decrease axial rotation. ${ }^{5}$ Frontal and horizontal curve correction is the aim of most brace systems, but sagittal plane correction, especially preservation of lumbar lordosis (LL), has been the focus since 2004 because of the development of sagittal plane measurement methods and the $3 \mathrm{D}$ reconstruction technique.

The aim of the present study was to retrospectively investigate progressive effects of the Chêneau brace on coronal plane, the sagittal plane, and pelvic parameters in patients with AIS.

ABBREVIATIONS AIS = adolescent idiopathic scoliosis; $\mathrm{LL}=$ lumbar lordosis; $\mathrm{PI}=$ pelvic incidence; $\mathrm{PT}=$ pelvic tilt; $\mathrm{SS}=$ sacral slope; $\mathrm{SVA}=$ sagittal vertical axis; $\mathrm{TK}=$ thoracic kyphosis.

SUBMITTED September 22, 2014. ACCEPTED February 3, 2015

INCLUDE WHEN CITING Published online July 10, 2015; DOI: 10.3171/2015.2.SPINE14970.

DISCLOSURE The authors report no conflict of interest concerning the materials or methods used in this study or the findings specified in this paper. 


\section{Methods \\ Patients}

The target population for this study was patients with AIS and with a very high risk of curve progression. Included were patients who were diagnosed with AIS and had a prescription for a Chêneau-type thoracolumbosacral orthosis, were aged $\geq 10$ years, were at Risser Stage 0-2, had an initial Cobb angle between $20^{\circ}$ and $40^{\circ}$, and, if female, were either premenarcheal or $<1$ year postmenarcheal. Patients who had been treated previously were excluded from the study. ${ }^{18}$ Each patient was treated with a Chêneau brace and given instructions to wear it for a minimum number of hours per day. ${ }^{3}$

Of the 326 patients in our institution who were treated with a brace for scoliosis between 2005 and 2012, 32 patients ( 29 girls and 3 boys) met our inclusion criteria. We excluded any patient who had early-onset AIS, who did not have anteroposterior and lateral radiographs available or whose images were unclear, who was treated with a different type of brace, or who was treated before the first radiographs were obtained. The average age at initiation of brace therapy was 12.9 years (range 11.1-14.5 years; SD 0.9 ), and the average duration of brace therapy was 24.4 months (range 12-69 months) (Table 1).

\section{Interventions and Data Collection}

Patients who were prescribed the brace were instructed to wear it for a minimum of 23 hours/day. Each patient was asked to undergo follow-up at 4- to 6-month intervals, at which times we collected radiographic, clinical, orthotic, and self-reported data. Follow-up was determined to be final when the first of 2 conditions was met: the curve had progressed to $\geq 50^{\circ}$ (treatment failure) or the patient reached skeletal maturity (Risser Stage 4 for females, Risser Stage $>4$ for males) without this degree of curve progression (treatment success). Treatment was considered complete when the patient had reached Risser Stage 4 (if female) or $>4$ (if male) and did not show any further growth according to length measurements. Information gathered from the medical records included age at diagnosis and at brace fitting, sex, history of treatments, menarcheal status, and duration of brace use.

\section{Radiology}

A standardized form was used to obtain data at initiation of brace therapy and at the last follow-up examination. Full-length, orthogonal anteroposterior and lateral radiographs were obtained simultaneously with the patient in a standardized standing position, with shoulder flexion to $30^{\circ}$, elbows fully flexed, and fists resting on the clavicles to avoid overlap with spinal structures. ${ }^{7}$ All radiological measurements were performed by 1 author (M.F.) using the same protractor and controlled by an experienced radiologist to minimize interobserver variability. The measurement error was within limits determined by the reproducibility of readings of repeated measurements of all radiographs from 10 patients at regular intervals. In patients with double-curved scoliosis, the larger curve before bracing was defined as the major curve.

Radiographic data at brace initiation and at the last
TABLE 1. Characteristics at baseline in 32 patients using a Chêneau brace*

\begin{tabular}{cc}
\hline Characteristic & Value \\
\hline Age at brace initiation (yrs) & $12.9 \pm 0.9$ \\
\hline Female sex & $29(91)$ \\
\hline SRS curve classification & $9(28)$ \\
\hline Thoracic & $6(19)$ \\
\hline Thoracolumbar & $11(34)$ \\
\hline Lumbar & $6(19)$ \\
\hline Double major & $14(44)$ \\
\hline Risser Stage & $10(31)$ \\
\hline 0 & $8(25)$ \\
\hline 1 & $24.4 \pm 12.5$ \\
\hline 2 &
\end{tabular}

SRS = Scoliosis Research Society.

${ }^{*}$ Values presented are mean $\pm \mathrm{SD}$ or number (\%).

follow-up without the brace were collected. We measured the Cobb angle, sagittal parameters (T4-T12 thoracic kyphosis [TK] angle, L1-S1 LL angle, and sagittal vertical axis [SVA]), and pelvic parameters (pelvic incidence [PI], sacral slope [SS], and pelvic tilt [PT]).

\section{Statistical Analysis}

The values of all aforementioned parameters were collected at brace initiation and at the last follow-up for each patient. For each parameter and each patient, the variation of the values was categorized as increased (the value at weaning increased $>5^{\circ}$ or $>20 \mathrm{~mm}$ over that at initiation), unchanged (the variation of the value at weaning was $<$ $5^{\circ}$ or $<20 \mathrm{~mm}$ over that at initiation), or decreased (the value at weaning decreased $>5^{\circ}$ or $>20 \mathrm{~mm}$ from that at initiation).

A statistical analysis was performed using SPSS version 19.0.1 for Windows. Mean values of each parameter were also compared at brace initiation and at the final follow-up by using a paired t-test. Differences between those two time points for each parameter according to the type of curvature were tested by using 1-way ANOVA adjusted for the least-significant difference. For each test, a p value of $<0.05$ was considered significant.

Approval for this study was obtained from the institutional review board of the Second Affiliated Hospital of Wenzhou Medical University.

\section{Results}

The differences in the values for each parameter before brace treatment and at final follow-up are shown in Table 2.

The Cobb angle decreased $>5^{\circ}$ in $16(50 \%)$ cases, remained unchanged in 10 (31\%), and increased in $6(19 \%)$. Among the 16 cases in which the Cobb angle decreased, it decreased $>10^{\circ}$ in $9(28 \%)$ cases. Lumbar lordosis decreased in 20 (63\%) cases, remained unchanged in 11 (34\%), and increased in $1(3 \%)$. Thoracic kyphosis decreased in 
TABLE 2. Variation in parameters between brace initiation and weaning

\begin{tabular}{|c|c|c|c|c|c|c|c|}
\hline \multirow[b]{3}{*}{ Variation* } & \multicolumn{7}{|c|}{ No. of Patients (\%) } \\
\hline & \multirow[b]{2}{*}{ Cobb Angle } & \multicolumn{2}{|c|}{ Sagittal Parameters } & \multicolumn{3}{|c|}{ Pelvic Parameters } & \multirow[b]{2}{*}{ Sagittal Balance } \\
\hline & & TK & LL & PT & $\mathrm{PI}$ & SS & \\
\hline Increased & $6(19)$ & $3(9)$ & $1(3)$ & $12(38)$ & $9(28)$ & $5(16)$ & $14(44)$ \\
\hline Unchanged & $10(31)$ & $8(25)$ & $11(34)$ & $16(50)$ & $19(59)$ & $16(50)$ & $14(44)$ \\
\hline Decreased & $16(50)$ & $21(66)$ & $20(63)$ & $4(12)$ & $4(13)$ & $11(34)$ & $4(12)$ \\
\hline
\end{tabular}

* A parameter was considered increased when the value at weaning increased $>5^{\circ}$ or $>20$ mm over that at initiation, unchanged when the value at weaning was $<5^{\circ}$ or $<20 \mathrm{~mm}$ over that at initiation, and decreased when the value at weaning decreased $>5^{\circ}$ or $>20 \mathrm{~mm}$ from that at initiation.

$21(66 \%)$ cases, remained unchanged in $8(25 \%)$, and increased in $3(9 \%)$. Pelvic incidence decreased in $4(13 \%)$ cases, remained unchanged in $19(59 \%)$, and increased in $9(28 \%)$. Pelvic tilt decreased in $4(12 \%)$ cases, remained unchanged in $16(50 \%)$, and increased in $12(38 \%)$. The SS decreased in 11 (34\%) cases, remained unchanged in 16 $(50 \%)$, and increased in $5(16 \%)$. The SVA moved forward (increased) in 14 (44\%) cases, remained unchanged in 14 (44\%), and moved backward (decreased) in $4(12 \%)$.

Differences in all parameters between brace initiation and the final follow-up are shown in Table 3. The mean Cobb angle improved from $30.6^{\circ}$ (SD 5.9 ${ }^{\circ}$ ) to $27.3^{\circ}$ (SD $\left.14.2^{\circ}\right)$, but no statistical difference was noted $(\mathrm{p}=0.13)$. The mean LL angle significantly decreased from $59.8^{\circ}$ (SD $9.9^{\circ}$ ) to $52.6^{\circ}$ (SD $8.8^{\circ}$ ). The mean TK angle significantly decreased from $28.0^{\circ}$ (SD 13.3 ${ }^{\circ}$ ) to $20.9^{\circ}$ (SD 13.0 $0^{\circ}$. The mean PT increased significantly $(\mathrm{p}=0.002)$ from $4.5^{\circ}$ (SD $8.7^{\circ}$ ) to $8.3^{\circ}\left(\mathrm{SD} 6.3^{\circ}\right)$, but no statistical difference was noted with the PI $(p=0.11)$ or SS $(p=0.23)$. The SVA was almost significantly $(\mathrm{p}=0.02)$ increased from $-44.0 \mathrm{~mm}$ (SD 39.1) to -30.2 $\mathrm{mm}$ (SD 28.5) at the final follow-up.

Differences in each parameter between the time of brace therapy initiation and the final follow-up according to the kind of curve are shown in Table 4. There were no significant differences in any of these parameters between brace initiation and the final follow-up according to the type of curve, except for the TK angle $(p=0.01)$. The double major curve had higher mean differences in TK angle values than in other kinds of curves.

\section{Discussion}

The immediate correction of AIS in the coronal, sagittal, and axial planes provided by the Chêneau brace has been well documented. ${ }^{11}$ The purpose of this study was to analyze the variability of the long-term effects of bracing on AIS. The main finding of the study is that the Chêneau brace induces a significant decrease in kyphosis, lordosis, and PT. In addition, it resulted in a significant shift forward of the SVA.

The main objective of brace treatment is to stabilize the shape of the scoliotic spine and prevent curve progression. At the final follow-up, the success rate of brace treatment in our study was $81 \%$. In the literature, success rates and follow-up durations associated with other braces were lower than those associated with the Chêneau brace, ${ }^{1,8,20,21}$ but the results of other published studies were not certain. The Chêneau brace resulted in a higher percentage of correction for thoracic curves at the final follow-up; however, it may not prevent curve progression effectively for double major curves. The results of the present study differed from those of De Giorgi et al., ${ }^{5}$ who reported a high percentage of correction for all curves and a more significant percentage of correction at the final follow-up for thoracolumbar curves. However, there may have been a bias in their study because of the small sample size. These results demonstrate that it is possible to achieve excellent prevention of curve progression, except for double major curves, if treatment is started at an appropriate time and initiated with an adequate brace.

Most of the previous clinical studies focused on the coronal plane. However, the other 3D parameters are of primary importance, because it is mandatory to stabilize the coronal plane without creating adverse effects on sagittal alignment and transverse plane parameters.

Many brace-related concepts have resulted in good correction but have had a deleterious effect on the sagittal profile, which may be related to the production of lumbar

TABLE 3. Correction of parameters with the Chêneau brace

\begin{tabular}{lcccrrr}
\hline \multicolumn{1}{c}{ Parameter } & Before Treatment* & At Final Follow-Up* & Difference $\dagger$ & $95 \% \mathrm{Cl}$ & t Value & $p$ Value \\
\hline Cobb angle $\left(^{\circ}\right)$ & 30.6 & 27.3 & $-3.3 \pm 12.0$ & -7.6 to 1.0 & -1.6 & 0.13 \\
\hline $\mathrm{TK}\left({ }^{\circ}\right)$ & 28.0 & 20.9 & $-7.1 \pm 10.8$ & -11.0 to 3.2 & -3.7 & $<0.01$ \\
\hline $\mathrm{LL}\left(^{\circ}\right)$ & 59.8 & 52.6 & $-7.1 \pm 8.2$ & -10.1 to -4.1 & -4.9 & $<0.01$ \\
\hline $\mathrm{PT}\left({ }^{\circ}\right)$ & 4.5 & 8.3 & $3.8 \pm 6.4$ & 1.5 to 6.1 & -3.4 & $<0.01$ \\
\hline $\mathrm{PI}\left({ }^{\circ}\right)$ & 45.0 & 47.3 & $2.3 \pm 7.9$ & -0.5 to 5.1 & 1.7 & 0.11 \\
\hline $\mathrm{SS}\left({ }^{\circ}\right)$ & 40.5 & 39.0 & $-1.5 \pm 6.9$ & -4.0 to 1.0 & -1.2 & 0.23 \\
\hline Sagittal balance $(\mathrm{mm})$ & -44.0 & -30.2 & $13.8 \pm 32.1$ & 2.2 to 25.3 & 2.4 & 0.02 \\
\hline * Value is presented as the mean. & & & & & &
\end{tabular}


TABLE 4. Type of curve and brace correction for each parameter

\begin{tabular}{|c|c|c|c|c|c|c|c|}
\hline Curve Type \& Statistics & Cobb Angle $\left({ }^{\circ}\right)$ & Kyphosis $\left({ }^{\circ}\right)$ & Lordosis $\left({ }^{\circ}\right)$ & $\mathrm{PT}\left({ }^{\circ}\right)$ & $\mathrm{PI}\left({ }^{\circ}\right)$ & $\operatorname{SS}\left({ }^{\circ}\right)$ & $\begin{array}{l}\text { Sagittal Balance } \\
(\mathrm{mm})\end{array}$ \\
\hline \multicolumn{8}{|l|}{ Curve type (mean \pm SD) } \\
\hline Thoracic & $-9.9 \pm 10.5$ & $-6.0 \pm 6.9$ & $-10.0 \pm 8.8$ & $2.8 \pm 4.8$ & $1.4 \pm 10.4$ & $-1.5 \pm 9.2$ & $-0.5 \pm 36.9$ \\
\hline Thoracolumbar & $-4.7 \pm 7.8$ & $-4.6 \pm 7.5$ & $-7.1 \pm 4.0$ & $5.1 \pm 6.1$ & $-0.8 \pm 5.7$ & $-5.9 \pm 3.3$ & $17.8 \pm 26.8$ \\
\hline Lumbar & $-2.3 \pm 9.7$ & $-2.6 \pm 8.2$ & $-2.5 \pm 8.2$ & $5.1 \pm 7.7$ & $4.4 \pm 8.2$ & $-0.7 \pm 5.9$ & $14.2 \pm 30.9$ \\
\hline Double major & $6.1 \pm 16.7$ & $-19.5 \pm 14.8$ & $-11.4 \pm 7.7$ & $1.7 \pm 6.7$ & $3.1 \pm 4.2$ & $1.5 \pm 6.7$ & $30.6 \pm 29.5$ \\
\hline \multicolumn{8}{|l|}{ Statistic } \\
\hline F value & 2.492 & 4.577 & 2.375 & 0.495 & 0.610 & 1.265 & 1.201 \\
\hline$p$ value & 0.081 & 0.010 & 0.091 & 0.689 & 0.614 & 0.305 & 0.328 \\
\hline
\end{tabular}

and thoracic flat back. The Chêneau system was designed with the principles of $3 \mathrm{D}$ correction in mind. The anterior pad pushes the lumbar and thoracic regions backward to fight posterior pressure on the rib hump and avoid flatback syndrome..$^{19}$ A significant diminution of TK and LL was observed in this study. In $66 \%$ of the cases, TK decreased, and in $63 \%$ of the cases, LL decreased. Seven (22\%) of the patients were found to have thoracic flat back, but 3 of them had it before treatment. A previous study by Courvoisier et al. ${ }^{4}$ on the immediate correction provided by brace treatment showed significant decreases in LL but no statistical difference in TK. However, progressive modifications of the spine may occur throughout the treatment period. An anterior pad was not enough to fight effectively against flat-back syndrome, especially at the thoracic region. Because of the posterior pressure on the rib hump, the SVA moved forward significantly and it had a positive effect on sagittal balance.

In our study, the brace-induced correction of pelvic parameters was also highly variable. The modification of PT varied; in 50\% of the cases, PT remained unchanged, and in $38 \%$ of the cases, it increased. However, when considering the global statistics, PT increased significantly from $4.5^{\circ}$ to $8.3^{\circ}$. Previous studies showed that the PI is a relatively fixed value. According to the reports of Mac-Thiong et al. and others, ${ }^{14-16}$ there is a slight tendency for PI to increase after the acquisition of bipedalism during growth until skeletal maturity, whereas SS remains relatively stable. In our study, PI and SS were unchanged in most of the cases and showed no significant differences between the time of the initiation of treatment and the time of final follow-up, which is consistent with the results of previous studies. The Chêneau brace has a pad at the pelvic level that confers pressure on the pelvis. For the cases in which PI changed, our hypothesis is that the diminution of PI occurs when a brace applies long-term pressure on the caudal aspect of the sacrum and at the anterior aspect of the iliac crest. Augmentation of the PI occurs when a brace applies long-term pressure on the cranial aspect of the sacrum and at the anterior aspect of the iliac crest. Significant diminution of LL was observed in our study, and it modified sagittal balance. To adapt to the modification of sagittal balance, the pelvis retroverted and PT increased. A previous study showed that LL was significantly correlated with PI and SS in AIS..$^{13}$ Chêneau brace treatment may influence this relationship and sagittal global balance.
There is still a lack of knowledge of the exact mechanisms of correction provided by brace treatment. In the current study, the suggested mechanisms are both an adaptation of the pelvis to modification of the sagittal balance and longterm compression of the pelvis by the brace.

Because of the limitations of radiographic modalities, we did not evaluate progressive correction of transverse plane parameters in this study. A previous study found no statistically significant immediate modification of transverse plane parameters. ${ }^{4}$ Lebel et al. ${ }^{11}$ compared the differences in immediate 3D effects between the Chêneau brace and the Boston brace by assessing 3D reconstructions. They demonstrated that the Chêneau brace had a significant de-rotation effect compared with the Boston brace. However, differences in immediate correction may not translate into differences in progressive correction. The long-term effect of this difference should be tested further in a prospective clinical trial by using 3D reconstruction technology.

Limitations of the present study included the small number of patients and measurements that were based on digital radiographs. A possible bias may have been present in the angular values. The expertise achieved by the brace maker was essential in obtaining good results, which could be considered another weakness of the study. The strengths of the study are that it was a homogeneous case series of patients with only one brace prescriber, only one brace maker, effective correction control assessed by radiographs inside the brace, and careful selection of patients with regard to brace wearing (scoring was based on answers from the patient and his or her parents to the questions: "Has the brace been used as prescribed? If not, do you use the brace at all?" and "For how many hours per week or per day are you not using the brace?"; patients were classified as compliant if the brace was used $>23$ hours/day until weaning).

\section{Conclusions}

The main purpose of the Chêneau brace is to provide 3D correction of AIS without sacrificing the sagittal profile. The results of our clinical study show that the Chêneau brace can be effective in preventing curve progression in patients with AIS. However, this study reveals the high variability of the effect of brace treatment on sagittal alignment. Chêneau brace treatment may influence 
sagittal global balance. Additional studies are needed to explain the mechanisms of 3D correction provided by the Chêneau brace.

\section{References}

1. Blaha J, Bukac J: [Correction of idiopathic scoliotic curves by means of conservative treatment using spinal bracing.] Acta Medica (Hradec Kralove) Suppl 48:31-35, 2005 (Czech)

2. Chêneau J (ed): Corset-Chêneau: Manuel d'Orthopédie des Scolioses suivant la Technique originale. Paris: FrisonRoche, 1994

3. Chêneau J: Scoliosis treating brace evaluation of our brace since 1970: An evaluation of the normalization of rotation, of rib static and of the wedge shaped vertebrae. Locomotor System 1:29-38, 2003

4. Courvoisier A, Drevelle X, Vialle R, Dubousset J, Skalli W: 3D analysis of brace treatment in idiopathic scoliosis. Eur Spine J 22:2449-2455, 2013

5. De Giorgi S, Piazzolla A, Tafuri S, Borracci C, Martucci A, De Giorgi G: Chêneau brace for adolescent idiopathic scoliosis: long-term results. Can it prevent surgery? Eur Spine J 22 (Suppl 6):S815-S822, 2013

6. Dolan LA, Weinstein SL: Surgical rates after observation and bracing for adolescent idiopathic scoliosis: an evidencebased review. Spine (Phila Pa 1976) 32 (19 Suppl):S91S100, 2007

7. Dubousset J, Charpak G, Skalli W, Kalifa G, Lazennec JY: [EOS stereo-radiography system: whole-body simultaneous anteroposterior and lateral radiographs with very low radiation dose.] Rev Chir Orthop Repar Appar Mot 93 (6 Suppl):141-143, 2007 (Fr)

8. Jonasson-Rajala E, Josefsson E, Lundberg B, Nilsson H: Boston thoracic brace in the treatment of idiopathic scoliosis. Initial correction. Clin Orthop Relat Res (183):37-41, 1984

9. Kotwicki T, Chêneau J: Biomechanical action of a corrective brace on thoracic idiopathic scoliosis: Cheneau 2000 orthosis. Disabil Rehabil Assist Technol 3:146-153, 2008

10. Landauer F, Wimmer C: Therapieziel der Korsettbehandlung bei idiopathischer Adoleszentenskoliose. Med Orthop Tech 123:33-38, 2003

11. Lebel DE, Al-Aubaidi Z, Shin EJ, Howard A, Zeller R: Three dimensional analysis of brace biomechanical efficacy for patients with AIS. Eur Spine J 22:2445-2448, 2013

12. Lenssinck ML, Frijlink AC, Berger MY, Bierman-Zeinstra SM, Verkerk K, Verhagen AP: Effect of bracing and other conservative interventions in the treatment of idiopathic scoliosis in adolescents: a systematic review of clinical trials. Phys Ther 85:1329-1339, 2005

13. Mac-Thiong JM, Labelle H, Charlebois M, Huot MP, de Guise JA: Sagittal plane analysis of the spine and pelvis in adolescent idiopathic scoliosis according to the coronal curve type. Spine (Phila Pa 1976) 28:1404-1409, 2003

14. Mac-Thiong JM, Labelle H, Roussouly P: Pediatric sagittal alignment. Eur Spine J 20 (Suppl 5):586-590, 2011

15. Mangione P, Gomez D, Senegas J: Study of the course of the incidence angle during growth. Eur Spine J 6:163-167, 1997

16. Marty C, Boisaubert B, Descamps H, Montigny JP, Hecquet $\mathrm{J}$, Legaye J, et al: The sagittal anatomy of the sacrum among young adults, infants, and spondylolisthesis patients. Eur Spine J 11:119-125, 2002

17. Negrini S, Minozzi S, Bettany-Saltikov J, Zaina F, Chockalingam N, Grivas TB, et al: Braces for idiopathic scoliosis in adolescents. Spine (Phila Pa 1976) 35:1285-1293, 2010

18. Richards BS, Bernstein RM, D'Amato CR, Thompson GH: Standardization of criteria for adolescent idiopathic scoliosis brace studies: SRS Committee on Bracing and Nonoperative Management. Spine (Phila Pa 1976) 30:2068-2077, 2005

19. Rigo M, Weiss HR: The Chêneau concept of bracing-biomechanical aspects. Stud Health Technol Inform 135:303319, 2008

20. von Deimling U, Wagner UA, Schmitt O: [Long-term effect of brace treatment on spinal decompensation in idiopathic scoliosis. A comparison of Milwaukee brace-Chêneau corset.] Z Orthop Ihre Grenzgeb 133:270-273, 1995 (Ger)

21. Weiss HR, Weiss GM: Brace treatment during pubertal growth spurt in girls with idiopathic scoliosis (IS): a prospective trial comparing two different concepts. Pediatr Rehabil 8:199-206, 2005

\section{Author Contributions}

Conception and design: Fang. Acquisition of data: Fang, Xiang. Analysis and interpretation of data: Fang, Wang, Xiang. Drafting the article: Fang, Tian. Critically revising the article: Xu, Tian. Reviewed submitted version of manuscript: Xu, Tian. Approved the final version of the manuscript on behalf of all authors: Xu. Statistical analysis: Fang, Wang, Lou. Administrative/technical/ material support: $\mathrm{Xu}$.

\section{Correspondence}

Hua-Zi Xu, Zhejiang Spine Research Center, Department of Orthopaedic Surgery, Second Affiliated Hospital of Wenzhou Medical University, 109 Xueyuanxi Rd., Wenzhou 325027, China.e-mail: spine-xu@163.com. 